\title{
EFEITOS DA CINESIOTERAPIA EM PACIENTES NO PÓS-OPERATÓRIO DE RECONSTRUÇÃO DO LIGAMENTO CRUZADO ANTERIOR (LCA): REVISÃO DE LITERATURA
}

\author{
Alisson Lourenço Freitas Noia ${ }^{1}$ \\ Sinvaldo Silva Alves ${ }^{2}$ \\ Carolina Menezes Couceiros de $\mathrm{Matos}^{3}$ \\ Eliane Nascimento Ribeiro Milcent ${ }^{4}$
}

RESUMO: O ligamento cruzado anterior (LCA) tem como principal função evitar a anteriorização da tíbia em relação ao fêmur nos exercícios de cadeia aberta. O objetivo desse estudo foi o de comparar os efeitos dos exercícios de cadeia aberta e fechada no pósoperatório de reconstrução do ligamento cruzado anterior (LCA). Trata-se de uma revisão bibliográfica, com 7 artigos contidos nos resultados, tendo como base de dados: Scielo, PubMed, PEDro e Bireme. Teve-se como mais importante critério de inclusão pacientes que utilizaram as cadeias cinéticas aberta e fechada como base de tratamento. Os exercícios realizados em cadeia cinética fechada (CCF) alcançaram maiores resultados em quase todas as variáveis quando comparados com exercícios em cadeia cinética aberta (CCA). $\mathrm{Na}$ reabilitação do pós-operatório de LCA, os efeitos dos exercícios em CCF são mais satisfatórios no que diz respeito ao alívio da dor, ganho de ADM, melhora da função e principalmente na proteção e preservação do neoligamento.

Palavras-chave: Cadeia cinética aberta. Cadeia cinética fechada. Ligamento cruzado anterior. LCA. Pós-operatório.

ABSTRACT: The main function of the anterior cruciate ligament (ACL) is to prevent the anteriorization of the tibia in relation to the femur in open-chain exercises. The aim of this study was to compare the effects of open and closed chain exercises in the postoperative period of anterior cruciate ligament (ACL). It is a bibliographic review, with 7 articles contained in the results, and had as database: Scielo, PubMed, PEDro and Bireme. The most important inclusion criterion was patients who used open and closed chains as a treatment basis. Exercises performed in closed kinetic chain (CKC) achieved greater results in almost all variables when compared to exercises in open kinetic chain (OKC). Therefore, it was observing than in the postoperative rehabilitation of the ACL the effects of exercises on $\mathrm{CKC}$ are more satisfactory with regard to pain relief, ROM gain, and improving function and especially in the protecting and preservation of neoligans.

\footnotetext{
I Pós-graduação em Fisioterapia Hospitalar com ênfase em Unidade de Terapia Intensiva de Alta Complexidade Adulto, Faculdade Santa Casa - Fisioterapeuta, Centro Universitário UnidomPedro.

${ }^{2}$ Fisioterapeuta, Centro Universitário UnidomPedro.

${ }^{3}$ Especialista em Unidade de Terapia Intensiva adulto, Faculdade Bahiana de Medicina e Saúde Pública

- Fisioterapeuta, UCSal - Docente do Centro Universitário UnidomPedro.

${ }^{4}$ Fisioterapeuta, Centro Universitário UnidomPedro.
} 
Keywords: Open kinetic chain. Closed kinetic chain. Anterior cruciate ligament. LCA. Postoperative.

\section{INTRODUÇÃO}

O joelho é um complexo intermediário do membro inferior formado pelas articulações femoropatelar e femorotibial, composto por estruturas ósseas, musculares e ligamentares, sendo que os sistemas muscular e ligamentar são os principais responsáveis por sua estabilização. O ligamento cruzado anterior (LCA) está situado na parte central e anterior da cápsula articular, porém, externamente à cavidade sinovial, tendo como principal função evitar a anteriorização da tíbia em relação ao fêmur nos movimentos de cadeia aberta. Os primeiros relatos de lesão dessa estrutura surgiram no século IX, entretanto, os primeiros achados de reconstrução cirúrgica vieram à tona somente no início do século $\mathrm{XX}$. (ABDALLA et al., 2009; PIEREZAN et al., 2017; SOARES, A. et al., 2017; SOARES, M. et al., 20II; SOUZA; TRIBIOLI, 20II).

A ruptura do LCA está geralmente ligada a uma rotação, a um salto, a uma desaceleração, ou a uma mudança de direção do movimento corporal associada a um giro com a extremidade do membro inferior fixa. A incidência deste evento é maior em indivíduos fisicamente ativos com idade entre 15 e 25 anos praticantes de atividades esportivas. Os estudos apontam que mais de roo.ooo procedimentos cirúrgicos são realizados anualmente nos Estados Unidos para a reconstrução desse ligamento. A instabilidade do joelho é comum após a ruptura do LCA, podendo evoluir para maiores déficits na funcionalidade, além do comprometimento de outras estruturas vizinhas. (BORIN et al., 2010; DAMBROS et al., 2012; FROBELL et al., 20I0; SOUZA; TRIBIOLI, 20II; VIDMAR et al., 2019).

Para a reconstrução do LCA, a eleição do melhor tipo de enxerto é tema para discussão. Ao longo do século XX, diversos cirurgiões realizavam reconstruções desse ligamento de forma experimental, utilizando enxertos através do tendão patelar, osso tendão osso (OTO), osso tendão (OT) e isquiotibiais (IT), contudo, atualmente, o enxerto osso tendão osso (OTO) é considerado padrão ouro para este tipo de procedimento. (BITUN et al., 2015; SALGADO; CASTRO, 2014). 
O enxerto através do OTO apresenta um bom prognóstico no que diz respeito à fixação e cicatrização, além de trazer como benefício uma boa estabilidade do joelho a longo prazo e uma reabilitação com maior intensidade. A revascularização deste novo ligamento tem início na segunda semana e o processo de ligamentizacão se dá em até dois anos após a cirurgia. A reconstrução traz como objetivo a devolução da estabilidade do joelho acometido e uma completa recuperação no que diz respeito à funcionalidade. (BIELER et al., 20I4; PEREIRA et al., 2012; SOUZA; TRIBIOLI, 20II; THIELE et al., 2009).

No pós-operatório de reconstrução do LCA, a literatura preconiza tratamento em cadeia cinética aberta (CCA) e cadeia cinética fechada (CCF). Em exercícios de CCA, o segmento distal do membro inferior (pé) se movimenta livremente no espaço, enquanto nos exercícios de CCF, o pé fica fixo em alguma estrutura que, por sua vez, gera movimento nos segmentos proximais (quadril e joelho). Alguns clínicos apontam que exercícios em CCF apresentam maior confiabilidade e funcionalidade em relação à CCA, apesar disso, recentes estudos apontam que exercícios em CCA apresentam maior eficiência no que se refere ao ganho de força muscular do quadríceps em relação à CCF. (KANG; JUNG; YU, 20I2; UÇAR et al., 2014).

O objetivo deste estudo foi comparar os efeitos dos exercícios de cadeia cinética aberta e fechada no Pós-operatório de reconstrução do ligamento cruzado anterior (LCA).

\section{METODOLOGIA}

O presente artigo trata-se de uma revisão bibliográfica, cujo levantamento do estudo foi realizado através das bases de dados Scielo, PubMed, PEDro e Bireme, no período entre Agosto de 2019 e Maio de 2020, onde foram inseridas publicações dos últimos dez anos que abordassem os efeitos das Cadeias Cinética Aberta (CCA) e fechada (CCF) em pacientes no pós operatório de LCA. Como estratégia de busca, utilizou-se as seguintes palavras-chaves com os seus respectivos correlacionados na língua estrangeira: cadeia cinética aberta, cadeia cinética fechada, ligamento cruzado anterior, LCA e pós-operatório. Também foram inseridos, em caráter excepcional, dois artigos do ano de 2007, por obterem informações de extrema relevância para a construção desta obra. Como critérios de inclusão pacientes com lesão do LCA, concomitante ou não a lesão de menisco, submetidos a 
tratamento em CCA e CCF, independentemente de raça, sexo, cor ou nacionalidade, pacientes com lesão unilateral e com idade entre I8 e 40 anos. Como critérios de exclusão, pacientes que passaram por cirurgias prévias no joelho, pacientes com lesão de menisco contralateral e pacientes com déficit cognitivo importante. Foram selecionados 30 artigos, 7 incluídos e excluídos 23 por não atenderem aos critérios dessa pesquisa.

\section{RESULTADOS}

Tabela I: resultados relacionados ao objetivo de comparar os efeitos dos exercícios de cadeia cinética aberta e fechada no Pós-operatório de reconstrução do ligamento cruzado anterior (LCA)

\begin{tabular}{|c|c|c|c|}
\hline AUTOR/ANO & OBJETIVO & MÉTODOS & RESULTADOS \\
\hline $\begin{array}{l}\text { PIZZATO et al., } \\
2007\end{array}$ & $\begin{array}{l}\text { Analisar o } \\
\text { comportamento da } \\
\text { frequência } \\
\text { mediana (Fmed) } \\
\text { em indivíduos } \\
\text { com lesão do LCA } \\
\text { durante contrações } \\
\text { isométricas em } \\
\text { exercícios de } \\
\text { cadeia cinética } \\
\text { aberta (CCA) e } \\
\text { fechada (CCF). }\end{array}$ & $\begin{array}{l}\text { Ensaio clínico } \\
\text { randomizado. } \\
\text { voluntários, } \\
\text { dividido em três } \\
\text { grupos: LCA e LAC- } \\
\text { C, cada um com ıo } \\
\text { pacientes com lesão } \\
\text { unilateral e o grupo } \\
\text { A controle, com } 20 \\
\text { voluntários sem } \\
\text { lesão. Submetidos a } \\
\text { um protocolo de } \\
\text { exercícios de (CCA) } \\
\text { e (CCF). Avaliaram } \\
\text { (Fmed). }\end{array}$ & $\begin{array}{l}\text { Em CCF, não } \\
\text { evidenciaram } \\
\text { diferenças } \\
\text { significativas para os } \\
\text { valores da Fmed em } \\
\text { relação aos diferentes } \\
\text { grupos estudados } \\
(p>0,05) .\end{array}$ \\
\hline $\begin{array}{l}\text { VASCONCELOS } \\
\text { et al., } 2007\end{array}$ & $\begin{array}{l}\text { Avaliar a } \\
\text { translação anterior } \\
\text { da tíbia (TAT), }\end{array}$ & $\begin{array}{l}\text { Ensaio clínico } \\
\text { randomizado. } 40 \\
\text { voluntários, }\end{array}$ & $\begin{array}{l}\text { Os valores } \\
\text { significativamente } \\
\text { maiores de TAT foram }\end{array}$ \\
\hline
\end{tabular}




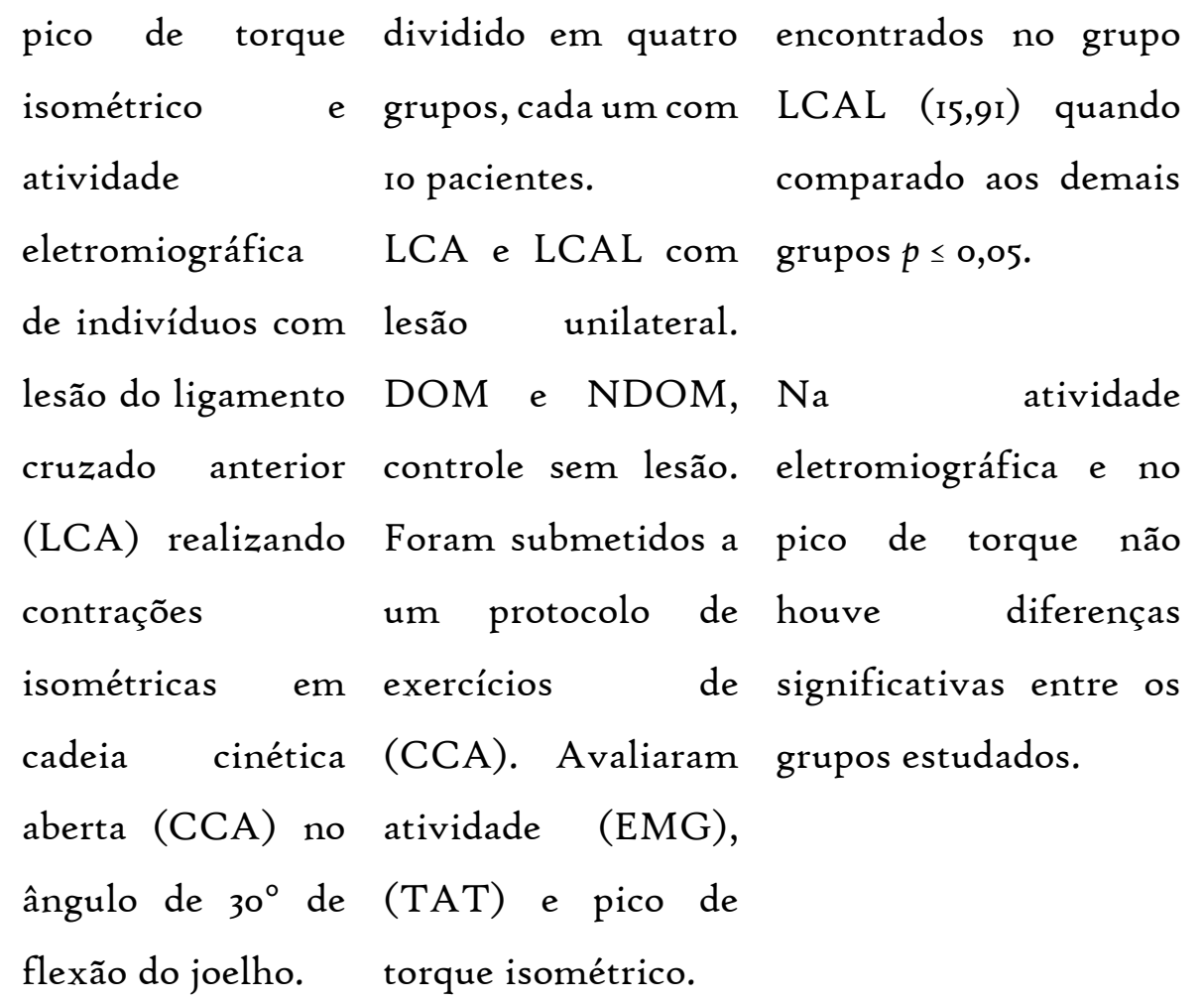

KANG; JUNG; Comparar a força e Estudo prospectivo, Após os exercícios YU, 2012

\begin{abstract}
resistência entre os controlado,
\end{abstract}
exercícios de randomizado.

cadeia cinemática 36 pacientes, com

aberta e fechada

após

a

reconstrução do

ligamento cruzado

anterior.
Lesão do LCA, diferenças

foram divididos em significativas para $o$

dois grupos: CKC e grupo OKC. Força 72,3

OKC, cada um com para $\uparrow$ II8,8, resistência I8 indivíduos, isocinética 46,9 para

realizaram $\uparrow 8 \mathrm{o}, 4$ e forças de

exercícios em CCF e agachamento de 136,I

CCA. Avaliaram para $\uparrow 164,7(\mathrm{p}<0,05)$.

força isocinética,

resistência e força de

agachamento. 
CHO; BAE; GAK, Examinar o efeito Ensaio clínico Em $15^{\circ}$ de 2013 dos exercícios de randomizado. 28 propriocepção não cadeia cinética pacientes com lesões houve diferenças entre fechada unilaterais do (LCA). os dois grupos, porém, executados por Distribuídos em dois em $45^{\circ}$ e nos escore de um grupo de grupos: SEG e UEG, Lysholm, o grupo exercício instável cada um com I4 UEG mostrou (UEG) e um pacientes realizaram diferenças grupo de exercício exercícios em CCF. significativas em estável (SEG) na Avaliaram relação ao SEG. De 4,3I articulação do propriocepção em $15^{\circ}$ para 2,80 e 48,oo para joelho, e $45^{\circ}$ e a função do 85,29 respectivamente propriocepção e joelho (escala de $(\mathrm{p}<0,05)$. escores funcionais Lysholm). de pacientes

submetidos à

reconstrução do

ligamento

cruzado anterior

(LCA).

FUKUDA Determinar se o Ensaio clínico Os grupos EOKC e et al., 2013 início precoce dos randomizado. 49 LOKC apresentaram exercícios da pacientes com lesões melhoras

CCA para força unilaterais do (LCA). significativas na do quadríceps em Distribuídos em dois função, na força e na uma $\quad$ ROM grupos: (EOKC) 25 diminuição da dor após restrita pacientes e $(\mathrm{LOKC})$ o tratamento. $(\mathrm{P}<0,05)$. promoveria uma 24 realizaram Contudo não houve melhora clínica exercícios de CCA. diferenças 


$$
\begin{array}{lrllr}
\text { sem causar } & \text { Avaliaram frouxidão } & \text { significativas entre os } \\
\text { aumento } & \text { da } & \text { anterior do joelho, grupos } & \text { quando } \\
\text { frouxidão anterior } & \text { força muscular do } & \text { comparados } & \text { em } \\
\text { do joelho em } & \text { quadríceps } & \text { e } & \text { nenhuma das variáveis } \\
\text { pacientes após a } & \text { isquiotibiais, função } & (\mathrm{P}>0,05) . & \\
\text { reconstrução do } & \text { e dor no joelho. }
\end{array}
$$

LCA.

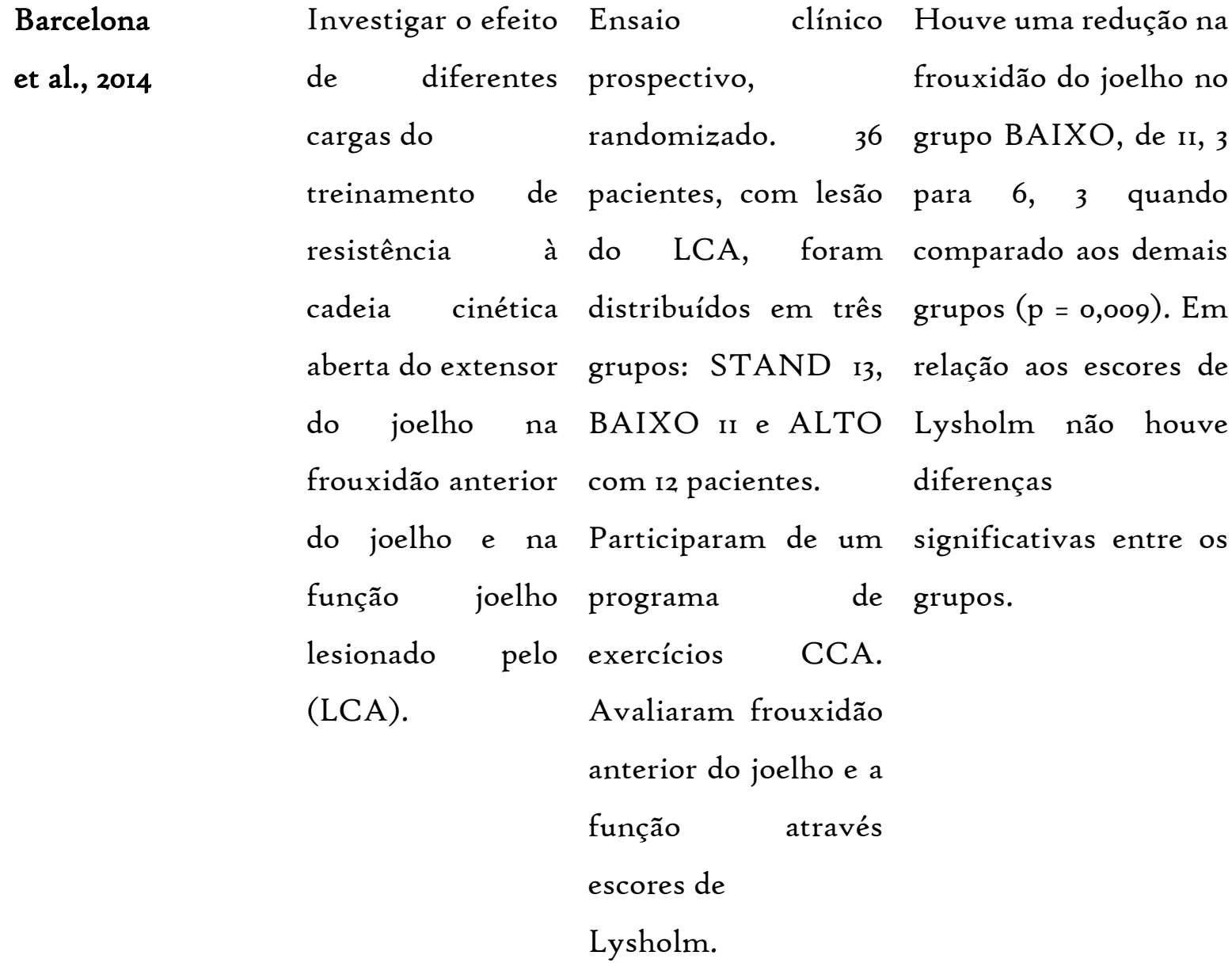

$\begin{array}{lllll}\text { UÇAR et al., } 2014 & \text { Comparar } & \text { os } & \text { Ensaio clínico } & \text { Ambos os grupos } \\ & \text { resultados } & \text { da } & \text { randomizado. } & \text { apresentaram } \\ & \text { reconstrução do } 58 \text { pacientes, divididos } & \text { melhoras nos escores, } \\ & \text { ligamento } & & \text { em } 2 \text { grupos: CKC } 30 & \text { porém, observou-se no } \\ & \text { cruzado anterior } & \text { pacientes e OKC } 28 . & \text { grupo CKC melhores }\end{array}$




$\begin{array}{llrlr}\text { após exercícios de } & \text { Avaliaram a } & \text { a índices em todas as } \\ \text { cadeia cinética } & \text { intensidade da dor variáveis quando } \\ \text { aberta e de cadeia } & \text { (EVA), circunferência comparado ao OKC (p } \\ \text { cinética fechada. } & \text { da coxa, ADM e a <0,05). } \\ & \text { função do joelho. }\end{array}$

Legenda: AD: Grupo controle dominante; ADM: amplitude de movimento; ALTO: Grupo treino com carga relativamente alta; AND: Grupo controle não-dominante; BAIXO: Grupo treino com carga relativamente baixa; CKC: Grupo que realizou exercícios em cadeia cinemática fechada; DOM: Grupo joelho dominante; EMG: Eletromiografia; EOKC: Grupo de exercícios com início precoce; Escala de Lysholm: Questionário de índice funcional da articulação do joelho, onde pontuações de zero a 6r, 62 a 8I, 82 a 90 e 9I a Ioo representam má, média, boa e excelente função do joelho, respectivamente; EVA: escala visual analógica; LCA: Grupo LCA lesado; LCA-C: Grupo Contralateral ao LCA lesado; LCAC: Grupo Ligamento cruzado colateral; LCAL: Grupo Ligamento cruzado anterior lesado; LOKC: Grupo de exercício com início tardio; NDOM: Grupo joelho não dominante; OKC: Grupo que realizou exercícios em cadeia cinemática aberta; ROM: Amplitude de movimento restrita; STAND: Grupo treino com protocolo padrão.

\section{DISCUSSÃO}

Pizzato et al. (2007) demonstraram valores da Fmed menores para indivíduos do grupo LCA. O grupo LCA, em CCA, demonstrou que os valores da frequência mediana foram significativamente menores nos músculos RC e VLL, quando comparado ao grupo LCA-C, e também obteve valores expressivamente inferiores quando comparado ao grupo A, desta vez para os músculos RC e BC. Observaram que o treinamento em CCF não tiveram modificações entre os grupos estudados no resultado de frequência mediana, e a CCA não demonstrou benefício satisfatório.

Vasconcelos et al. (2007) observaram que, tanto o Teste máxima manual quanto o Teste ativo do quadríceps modificado demonstraram valores da TAT superiores no grupo LCAL, quando comparado aos demais grupos. Após este resultado, foi feito um comparativo 
entre os demais grupos, porém não houve diferença significativa entre eles. Discordando de Pizzato et al. (2007), pois encontraram que na atividade eletromiográfica, não houve diferença estatisticamente significativa para os músculos do quadríceps e dos isquiotibiais em nenhum grupo avaliado.

Kang, Jung e Yu (2012) observaram que os indivíduos que realizaram exercícios em CCA, alcançaram melhores resultados na força e na resistência isocinética dos músculos flexores e extensores, além da melhora da força de agachamento, quando comparados aos que realizaram exercícios em CCF. Corroborando com Fukuda et al. (2013), que observaram que as variáveis dor, força do quadríceps e função do joelho foram melhoradas ao longo do tratamento, tanto para o grupo de início precoce quanto para o grupo de início tardio submetidos à CCA. A força dos isquiotibiais só tiveram benefícios significativos após tempo mais tardio, porém não houve diferença estatisticamente significativa na comparação entre os grupos para todas as avaliações funcionais e da dor, incluindo frouxidão do joelho.

Barcelona et al. (2014) demonstraram que o grupo que se utilizou da carga baixa alcançou maior diminuição da frouxidão do joelho em relação aos demais. Em relação aos valores dos escores de Lisholm, não houve diferença significativa entre os grupos estudados. Discordando de Fukuda et al. (2013), que ao observarem que após alcançarem resultados satisfatórios em ambos os grupos nas variáveis: dor, força e função, não houve aumento na frouxidão do joelho quando utilizaram exercícios em CCA.

Cho, Bae e Gak (2013) observaram que na avaliação clínica a $15^{\circ}$ de propriocepção não mostraram diferenças estatisticamente significantes entre os dois grupos. O grupo de exercício instável (UEG) e o grupo de exercício estável (SEG) exibiu diferenças estatisticamente significantes nas funções proprioceptivas em $15^{\circ}$ e escala de Lysholm e só após 6 semanas os dois grupos apresentaram diferença estatisticamente significante nas funções proprioceptivas a $45^{\circ}$.

Uçar et al. (2014), após compararem os resultados, observaram grande redução do índice de dor, diminuição da circunferência da coxa, aumento da flexão do joelho e dos escores da escala de Lysholm aos 3 e 6 meses após a cirurgia. Os exercícios realizados em CCF alcançaram maiores resultados em todas as variáveis quando comparados com 
exercícios em CCA, exceto a circunferência da coxa, que em ambos os grupos tiveram resultados equivalentes positivamente.

Cho, Bae e Gak (2013) e Uçar et al. (2014) corroboram quando afirmam que em seus estudos encontraram resultados positivos relacionados à escala de Lysholm utilizando a CCF.

\section{CONSIDERAÇÕES FINAIS}

Exercícios em CCA e CCF proporcionam efeitos benéficos para os pacientes com lesão do LCA, entretanto deve-se entender o mecanismo de cada uma delas para obtenção de melhores resultados no tratamento do pós-operatório. Com base nos estudos que compuseram esta obra, observou-se que na reabilitação do pós-operatório de LCA, os efeitos dos exercícios em CCF são mais satisfatórios quando comparados aos da CCA no que diz respeito ao alívio da dor, ganho de $\mathrm{ADM}$, melhora da função e principalmente na proteção e preservação deste neoligamento, pois exercícios em CCF evitam a translação anterior da tíbia que é um movimento prejudicial para o enxerto do LCA. Os exercícios em CCA devem ser inseridos de forma cautelosa e, portanto, numa fase mais avançada da reabilitação com intuito de fortalecer um grupamento muscular mais específico.

Se faz necessário novos estudos relacionados a este tema para confirmar os achados e fortalecer a teoria empregada.

\section{REFERÊNCIAS}

ABDALLA, R. J. et al. Comparação entre os resultados obtidos na reconstrução do ligamento cruzado anterior do joelho utilizando dois tipos de enxertos autólogos: tendão patelar versus semitendíneo e grácil. Rev Bras Ortop., v. 44, n. 3, p. 204-207, Maio./Jun. 2009. Disponível em: 〈https://www.scielo.br/j/rbort/a/qVYtjTqbwnzvsQBGkPSLzmf/?lang=pt〉. Acesso em: 2I mar. 2020.

BARCELLONA, M. G. et al. The effect of knee extensor open kinetic chain resistance training in the ACL-injured knee. Knee Surg Sports Traumatol Arthrosc., v. 23, n. II, p. 
3168-3177, Jun. 2014. Disponível em: 〈https://pubmed.ncbi.nlm.nih.gov/24934926/〉. Acesso em: 28 ago. 2019 .

BIELER, T. et al. The Effects of High-Intensity versus Low-Intensity Resistance Training on Leg Extensor Power and Recovery of Knee Function after ACL-Reconstruction. BioMed Research International, v. 2014, n. 278512, p. I-II, Abr. 2014. Disponível em: 〈https://www.hindawi.com/journals/bmri/2014/278512/>. Acesso em: nov. de 2019.

BITUN, P. B. et al. Comparação dos enxertos para reconstrução anatômica do LCA: patelar versus semitendíneo/grácil. Rev Bras Ortop. v. 50, n. I, p. 50-56, Jan. 2015. Disponível em: 〈https://www.scielo.br/j/rbort/a/jn8z7VvxjL3n5Rnnm9mhq5b/?format=pdf\&lang=pt . Acesso em: 17 maio 2020.

BORIN, G. et al. Controle postural em pacientes com lesão do ligamento cruzado anterior. Fisioterapia e Pesquisa, São Paulo, v.17, n.4, p. 342-345, Out./Dez. 2010. Disponível em: $\langle$ https://www.scielo.br/j/fp/a/rrbmJ8gnN6Qjy48kt4CLR3c/?lang=pt $\rangle$. Acesso em: Is fev. 2020.

CHO, S. H.; BAE, C. H.; GAK, H. B. Effects of closed kinetic chain exercises on proprioception and functional scores of the knee after anterior cruciate ligament reconstruction. J. Phys. Ther. Sci., v. 25, n. Io, p. 1239-1241, Abr./Maio 2013. Disponível em: 〈https://www.ncbi.nlm.nih.gov/pmc/articles/PMC3820198/>. Acesso em: 26 ago. 2019.

DAMBROS, C. et al. Effectiveness of cryotherapy after anterior Cruciate ligament reconstruction. Acta Ortop Bras, v. 20, n. 5, p. 285-290, Mar./Abr 2012. Disponível em: 〈https://www.ncbi.nlm.nih.gov/pmc/articles/PMC3718449/〉. Acesso em: 02 set. 2019.

FROBELL, R. B. et al. A Randomized Trial of Treatment for Acute Anterior Cruciate Ligament Tears. New England Journal of Medicine, v. 363, n. 4, p. 331-342, Jul. 2010. Disponível em: 〈https://www.nejm.org/doi/full/ı.1056/nejmoao907797〉. 23 jan. 2020. 
FUKUDA, T. Y. et al. Open Kinetic Chain Exercises in a Restricted Range of Motion After Anterior Cruciate Ligament Reconstruction: A Randomized Controlled Clinical Trial. The American Journal of Sports Medicine, v. 4I, n. 4, p. 788-794, Abr. 2013.

KANG, H.; JUNG, J.; YU, J. Comparison of Strength and Endurance between Open and Closed Kinematic Chain Exercises after Anterior Cruciate Ligament Reconstruction: Randomized Control Trial. J. Phys. Ther. Sci., v. 24, n. Io, p. 1055-1057, Maio./Jun. 2012.

PEREIRA, M. Tratamento fisioterapêutico após reconstrução do ligamento cruzado anterior. Acta ortop. bras., São Paulo, v. 20, n. 6, p. 371-375, Nov./Dez. 2012. Disponível em: 〈https://www.scielo.br/j/aob/a/9p7tXsSZCyQ7zLNQbYvyvcL/?lang=pt〉. Acesso em: 29 set. 2019.

PIEREZAN, B. et al. Análise do perfil oxidativo de diferentes amostras biológicas de pacientes com lesão de ligamento cruzado anterior. Fisioter Pesqui, v. 24, n. 2, p. 198-204, Abr./Jun. 2017. Disponível em: $\langle$ https://www.scielo.br/j/fp/a/TPC6CmBsbHdtg36rTDL7ssC/?lang=pt $>$. Acesso em: II maio 2020 .

PIZZATO, L. M. et al. Análise da freqüência mediana do sinal eletromiográfico de indivíduos com lesão do ligamento cruzado anterior em exercícios isométricos de cadeia cinética aberta e fechada. Rev Bras Med Esporte, v. 13, n. I, p. I-5, Jan./Fev. 2007. Disponível em: 〈https://www.scielo.br/j/rbme/a/bmBHP 4 BnZGvq95gdtBSY 3 dL/?lang=pt $>$. Acesso em: I8 jan. 2020.

SALGADO, J.; CASTRO, J. Ligamentoplastia do ligamento cruzado anterior com enxerto osso-tendão-osso vs enxerto de tendões isquiotibiais vs enxerto osso-tendão. Rev Port Ortop Traum., v. 22, n. 3, p. 265-283, Set. 2014. Io out. 2019. 
SOARES, A. C. et al. Qualidade de vida e capacidade funcional no pós-operatório tardio de ligamento cruzado anterior em trabalhadores de uma empresa de entrega de correspondências. Revista Unilago, v. I, n. I, p. I-8, 2017. Disponível em: $\langle$ http://revistas.unilago.edu.br/index.php/revista-medicina/article/view/rg〉. Acesso em: 13 abr. 2020.

SOARES, M. S. et al. Intervenção fisioterapêutica no pós-operatório de lesões do ligamento cruzado anterior. Revista Tem@, Campina Grande, v. II, n. I6, p. I-II, Jan./Jun. 2oIr. Disponível em: 〈http://revistatema.facisa.edu.br/index.php/revistatema/article/view/8I>. Acesso em: I5 dez. 2019.

SOUZA, K. T. M.; TRIBIOLI, M. Fisioterapia em lesão de ligamento cruzado anterior com ênfase no tratamento pós-operatório. Fisioterapia Brasil, v. I2, n. I, p. 47-52, Jan./Fev. 2011. Disponível em: 〈https://portalatlanticaeditora.com.br/index.php/fisioterapiabrasil/article/view/6oo〉. Acesso em: 24 nov. 2019.

THIELE, E. Protocolo de reabilitação acelerada após reconstrução de ligamento cruzado anterior - dados normativos. Rev. Col. Bras. Cir, v. 36, n. 6, p. 504-508, Nov./Dez. 2009. Disponível em: 〈https://www.scielo.br/j/rcbc/a/SQVdc6ghkYQRsJJbZN $49{ }_{43}$ s/abstract/?lang=en〉. Acesso em: 07 out. 2019.

UÇAR, M. et al. Evaluation of Open and Closed Kinetic Chain Exercises in Rehabilitation Following Anterior Cruciate Ligament Reconstruction. J. Phys. Ther. Sci., v. 26, n. I2, p. 1875-1878, Dez. 2014. Disponível em: 〈https://www.ncbi.nlm.nih.gov/pmc/articles/PMC4273046/〉. Acesso em: 19 fev. 2020.

VASCONCELOS, R. A. et al. Análise da translação anterior da tíbia, pico de torque e atividade eletromiográfica do quadríceps e isquiotibiais em indivíduos com lesão do ligamento cruzado anterior em cadeia cinética aberta. Acta Ortop. Bras., v. 15, n. I, p. I4-I8, 
Jul. 2007 . 〈https://www.scielo.br/j/aob/a/QsKzCFC5tX5Mj4ZTHs5xrnB/abstract/?lang=pt〉. Acesso em: 22 out. 2019.

VIDMAR, M. F. et al. Isokinetic eccentric training is more effective than constant load eccentric training on the quadriceps rehabilitation following anterior cruciate ligament reconstruction: a randomized controlled trial. Braz J Phys Ther, v. 39, p. 120-125, Set. 2019. Disponível em: 〈https://www.sciencedirect.com/science/article/abs/pii/SI413355519300097?via\%3Dihub〉. Acesso em: 19 ago. 2019. 